\title{
Dissipativity Analysis and Synthesis for a Class of Nonlinear Stochastic Impulsive Systems
}

\author{
Guici Chen, ${ }^{1,2}$ Jianzhong Zhou, ${ }^{1}$ and Yongchuan Zhang ${ }^{1}$ \\ ${ }^{1}$ School of Hydropower \& Information Engineering, Huazhong University of Science and Technology, Wuhan 430074, China \\ ${ }^{2}$ Hubei Province Key Lab of Systems Science in Metallurgical Process, Wuhan University of Science and Technology, \\ Wuhan 430081, China
}

Correspondence should be addressed to Guici Chen; gcichen@aliyun.com

Received 1 August 2013; Accepted 6 September 2013

Academic Editor: Tao Li

Copyright (C) 2013 Guici Chen et al. This is an open access article distributed under the Creative Commons Attribution License, which permits unrestricted use, distribution, and reproduction in any medium, provided the original work is properly cited.

\begin{abstract}
The dissipativity analysis and control problems for a class of nonlinear stochastic impulsive systems (NSISs) are studied. The systems are subject to the nonlinear disturbance, stochastic disturbance, and impulsive effects, which often exist in a wide variety of industrial processes and the sources of instability. Our aim is to analyse the dissipativity and to design the state-feedback controller and impulsive controller based on the dissipativity such that the nonlinear stochastic impulsive systems are stochastic stable and strictly $(Q, S, R)$-dissipative. The sufficient conditions are obtained in terms of linear matrix inequality (LMI), and a numerical example with simulation is given to show the correctness of the derived results and the effectiveness of the proposed method.
\end{abstract}

\section{Introduction}

As we all know that many real-world systems may be disturbed by stochastic factors. Thus, stochastic differential systems appear as a natural description of many observed phenomena of real world. In the past few years, much research effort has paid to the stability analysis and robust control problems for stochastic systems which have been come to play an important role in many fields including population dynamics, macroeconomics, chemical reactor control, communication network, image processes, and mobile robot localization. So far, plenty of significant results also have been published; see, for example, [1-8] and the references therein.

Recent years, there are many real-world systems and natural processes which display some kind of dynamic behavior in a style of both continuous and discrete characteristics; we called "impulsive effects," which exist widely in many evolution process, particularly some biological system such as biological neural networks and bursting rhythm models in pathology as well as optimal control models in economics, frequency-modulated signal processing system, fly object motions, and so on $[9,10]$. Impulsive dynamical systems are characterized by the occurrence of abrupt changes in the state of the system occurring at certain time instants. The stability and control problems of impulsive dynamical systems have attracted considerable interest in science and engineering during the past decades including stability analysis of stochastic impulsive systems or with time delay [1114], robust control of impulsive systems with time delay [15, 16], and robust control and filtering of stochastic impulsive systems with time delay [17].

On the other hand, since the notation of dissipative dynamical system was introduced by Willems [18], dissipative systems have been of particular interest to researchers in areas of systems, circuits, networks, control, and so forth. Now, dissipative theory has wide-ranging implications and applications in control theory. For instance, dissipativeness was crucially used in the stability analysis of nonlinear system [19], and the theory of dissipative systems generalizes basic tools including the passivity theory, bounded real lemma, Kalman Yakubovich lemma, and the circle criterion [20]. Among the relevant topics are the passivity analysis and synthesis for time-delay systems [21, 22]. These results show that the passivity-based methods are highly effective in designing the robust controller. 
The stability and stabilization [11-13, 19, 23], controllability [14], robust control $[15,16,20,22]$, robust filtering [17], and reliable dissipative control [24] problems have been extensively studied. However, the dissipative analysis and synthesis for nonlinear stochastic impulsive system have not been fully discussed and remains challenging. In this paper, we mainly study the dissipativity analysis and control problems for a class of nonlinear stochastic impulsive systems (NSISs). The systems are subject to the nonlinear disturbance, stochastic disturbance, and impulsive effects, which often exist in a wide variety of industrial processes and the sources of instability. Here, our aim is to analysis the dissipativity and to design the state-feedback controller and impulsive controller based on the dissipativity such that the nonlinear stochastic impulsive systems are stochastic stable and strictly $(Q, S, R)$-dissipative.

Notations. Throughout this paper, if not explicitly stated, matrices are assumed to have compatible dimensions. For symmetric matrices $X$ and $Y$, the notation $X \geq Y$ (resp., $X>$ $Y$ ) means that the matrix $X-Y$ is positive semidefinite (resp., positive definite). $I$ is a identity matrix with appropriate dimensions; the subscript " $T$ " represents the transposition. $E(\cdot)$ denotes the expectation operator with respect to some probability measure $P . L_{2}[0, \infty)$ is the space of square integrable vector functions over $[0, \infty)$; let $(\Omega, F, P)$ be a complete probability space which relates to an increasing family $\left(F_{t}\right)_{t>0}$ of $\sigma$ algebras $\left(F_{t}\right)_{t>0} \subset F$, where $\Omega$ is the samples space, $F$ is $\sigma$ algebra of subsets of the sample space, and $P$ is the probability measure on $F . R^{n}$ and $R^{n \times m}$ denote the $n$ dimensional Euclidean space and the set of all $n \times$ $m$ real matrices, respectively. For any $0<T<\infty$, we write $[0, T]$ for the closure of the open interval $(0, T)$ in $R$ and denote by $L_{2}^{n}\left([0, T] ; L^{2}\left(\Omega, K^{k}\right)\right)$ the space of the nonanticipative stochastic processes $y(\cdot)=(y(\cdot))_{t \in[0, T]}$ with respect to $\left(F_{t}\right)_{t \in[0, T]}$ satisfying $\|y(\cdot)\|_{L_{2}^{n}}^{2}=E\left(\int_{0}^{T}\|y(t)\|^{2} \mathrm{~d} t\right)=$ $\int_{0}^{T} E\left(\|y(t)\|^{2}\right) \mathrm{d} t<\infty . \lambda_{\min }(\cdot)$ and $\lambda_{\max }(\cdot)$ describe the minimum and maximum eigenvalue, respectively.

\section{A Class of Nonlinear Stochastic Impulsive Systems}

In this paper, we mainly consider the following nonlinear stochastic impulsive systems (NSISs):

$$
\begin{gathered}
\mathrm{d} x(t)=[A x(t)+B u(t)+f(t, x(t))+D v(t)] \mathrm{d} t \\
+\sigma(t, x(t)) \mathrm{d} w(t), \quad t \in\left(t_{k-1}, t_{k}\right], \\
\Delta x\left(t_{k}^{+}\right)=\left(E_{k}+\bar{E}_{k}\right) x\left(t_{k}\right), \quad t=t_{k}, \\
z(t)=C x(t), \\
x\left(t_{0}^{+}\right)=x_{0},
\end{gathered}
$$

where $x(t) \in R^{n}$ is the system state, $u(t) \in R^{l}$ is the control input, $v(t) \in R^{q}$ is the exogenous disturbance input of the systems which belong to $L_{2}[0, \infty), z(t) \in R^{r}$ is the system control output, and $w(t)$ is a zero mean real scalar Weiner processes on a probability space $(\Omega, F, P)$ relative to an increase family $\left(F_{t}\right)_{t>0}$ of $\sigma$ algebras $\left(F_{t}\right)_{t>0} \subset F$. A, B, C, and $D$ are the known real constant matrices with appropriate dimensions. Moreover, we assume that

$$
E(\mathrm{~d} w(t))=0, \quad E\left((\mathrm{~d} w(t))^{2}\right)=\mathrm{d} t .
$$

The sequence, $\left\{t_{k}, E_{k} x\left(t_{k}\right)\right\}$ and $\left\{t_{k}, \bar{E}_{k} x\left(t_{k}\right)\right\}$ in the systems describe the impulsive effect and impulsive control, respectively, where $E_{k}, \bar{E}_{k}$ are known real-valued matrices with appropriate dimensions, which have the effect of suddenly changing the state of the system at the point $t_{k} . \Delta x\left(t_{k}\right)=$ $x\left(t_{k}^{+}\right)-x\left(t_{k}^{-}\right)$, where $x\left(t_{k}^{-}\right)=\lim _{h \rightarrow 0^{+}} x\left(t_{k}-h\right), x\left(t_{k}^{+}\right)=$ $\lim _{h \rightarrow 0^{+}} x\left(t_{k}+h\right)$ with discontinuity instants $t_{0}<t_{1}<t_{2}<$ $\cdots<t_{k}<\cdots, \lim _{k \rightarrow \infty} t_{k}=\infty$; for convenience, let $t_{0}=0$ and $h>0$ be sufficiently small. Without loss of generality, it is assumed that $x\left(t_{k}\right)=x\left(t_{k}^{-}\right)=\lim _{h \rightarrow 0^{+}} x\left(t_{k}-h\right)$.

$f(\cdot, \cdot): R_{+} \times R^{n} \rightarrow R^{n}$ is an unknown nonlinear function which describes the system nonlinearity satisfying the following condition:

$$
f(0,0)=0, \quad\|f(t, x(t))\| \leq \beta\|x(t)\|,
$$

for a positive constant $\beta$. Equivalently stated, condition (3) implies that there exists a scalar $\kappa>0$ such that

$$
\kappa\left(\beta^{2} x^{T}(t) x(t)-f^{T}(t, x(t)) f(t, x(t))\right) \geq 0 .
$$

$\sigma(\cdot, \cdot): R_{+} \times R^{n} \rightarrow R^{n}$ also is an unknown nonlinear function which describes the stochastic nonlinearity satisfying

$$
\sigma^{T}(t, x(t)) \sigma(t, x(t)) \leq x^{T}(t) G^{T} G x(t)
$$

where $G$ is a known real constant matrices with approximate dimension.

\section{Preliminaries}

In this section, some definitions and lemmas are given.

Definition 1. For systems in (1) with $v(t)=0$, if there exists a constant $c>0$ satisfying $E \int_{0}^{\infty}\|x(t)\|^{2} \mathrm{~d} t \leq c\left\|x_{0}\right\|^{2}, x_{0} \in R^{n}$, systems in (1) are said to be stochastically stable.

Before giving the following definition, we firstly give the definition of quadratic energy supply function associated with systems in (1) as follows:

$$
\begin{aligned}
& \Psi(v, z, T) \\
& \quad=E \int_{0}^{T}\left(z^{T}(t) Q z(t)+2 v^{T}(t) S z(t)+v^{T}(t) R v(t)\right) \mathrm{d} t,
\end{aligned}
$$

where $Q, S$, and $R$ are real matrices with appropriate dimensions and $Q$ and $R$ are symmetrical. 
Definition 2. Systems in (1) are called $(Q, S, R)$-dissipative if for any $T>0$, under zero initial state, the following condition is satisfied:

$$
\Psi(v, z, T) \geq 0, \quad \forall T \geq 0 .
$$

Furthermore, if for a scalar $\alpha>0$, such that

$$
\Psi(v, z, T) \geq \alpha E \int_{0}^{T} v^{T}(t) v(t) \mathrm{d} t, \quad \forall T \geq 0,
$$

systems in (1) are called strictly $(Q, S, R)$-dissipative.

Lemma 3 (Schur complement lemma (see [25])). For a given matrix $S=\left(\begin{array}{cc}S_{1} & S_{3} \\ * & S_{2}\end{array}\right)$ with $S_{1}^{T}=S_{1}, S_{2}^{T}=S_{2}$, then the following conditions are equivalent:

$$
\begin{aligned}
& \text { (1) } S<0 \\
& \text { (2) } S_{2}<0, S_{1}-S_{3} S_{2}^{-1} S_{3}^{T}<0 \\
& \text { (3) } S_{1}<0, S_{2}-S_{3} S_{1}^{-1} S_{3}^{T}<0
\end{aligned}
$$

Lemma 4 (see [26]). For any $x \in R^{n}$, if $P \in R^{n \times n}$ is a positive definite matrix, $Q \in R^{n \times n}$ is a symmetric matrix, then

$$
\lambda_{\min }\left(P^{-1} Q\right) x^{T} P x \leq x^{T} Q x \leq \lambda_{\max }\left(P^{-1} Q\right) x^{T} P x .
$$

In this paper, our aim is to develop dissipativity criteria for systems in (1) based on Definition 2 and subsequently design the feedback dissipative controller $u(t)=K x(t)$.

\section{Main Results and Proofs}

4.1. Dissipativity Analysis of NSISs. For dissipativity analysis for NSISs, we treat the free systems as follows:

$$
\begin{gathered}
\mathrm{d} x(t)=[A x(t)+f(t, x(t))+D v(t)] \mathrm{d} t \\
+\sigma(t, x(t)) \mathrm{d} w(t), \quad t \in\left(t_{k-1}, t_{k}\right], \\
\Delta x\left(t_{k}^{+}\right)=\left(E_{k}+\bar{E}_{k}\right) x\left(t_{k}\right), \quad t=t_{k}, \\
z(t)=C x(t), \\
x\left(t_{0}^{+}\right)=x_{0} .
\end{gathered}
$$

Theorem 5. Given a real matrix $S$, the positive matrices $R, Z>$ 0 and a negative semidefinite matrix $Q \leq 0$, if there exist a scalar $\delta>0$ and a positive definite matrix $P>0$, such that the following inequalities hold:

$$
\begin{gathered}
\Xi=\left(\begin{array}{ccc}
\Delta & P D-C^{T} S & P \\
* & -R & 0 \\
* & * & -\kappa I
\end{array}\right)<0, \\
\beta_{k}=\lambda_{\max }\left(P^{-1}\left(I+E_{k}+\bar{E}_{k}\right)^{T} P\left(I+E_{k}+\bar{E}_{k}\right)\right)<1,
\end{gathered}
$$

where $\Delta=A^{T} P+P A+G^{T} P G+\kappa \beta^{2} I-C^{T} Q C+\delta Z$. Then the NSISs in (10) are stochastic stable and strictly $(Q, S, R)$ dissipative.
Proof. Construct a simple Lyapunov function $V(\cdot, \cdot): R^{n} \times$ $R_{+} \rightarrow R_{+}$as follows:

$$
V(x(t), t)=x^{T}(t) P x(t) .
$$

For $t \in\left(t_{k-1}, t_{k}\right], k=1,2, \ldots$, according to the Itô formula, along with the solution of systems in (10), we have

$$
\begin{aligned}
L V(x(t), t)= & 2 x^{T}(t) P[A x(t)+f(t, x(t))+D v(t)] \\
& +\sigma^{T}(t, x(t)) P \sigma(t, x(t)) .
\end{aligned}
$$

Applying (3)-(5), we get that

$$
\begin{aligned}
L V(x(t), t)= & x^{T}(t)\left(A^{T} P+P A\right) x(t)+2 x^{T}(t) P f(t, x(t)) \\
& +2 x^{T}(t) P D v(t)+\sigma^{T}(t, x(t)) P \sigma(t, x(t)) \\
\leq & x^{T}(t)\left(A^{T} P+P A+G^{T} P G+\kappa \beta^{2} I\right) x(t) \\
& +2 x^{T}(t) P f(t, x(t))+2 x^{T}(t) P D v(t) \\
& -k f^{T}(t, x(t)) f(t, x(t)) \\
= & \chi^{T}(t) \Theta \chi(t),
\end{aligned}
$$

where

$$
\begin{gathered}
\chi(t)=\left(\begin{array}{ccc}
x^{T}(t) & v^{T}(t) & f^{T}
\end{array}\right)^{T}, \\
\Theta=\left(\begin{array}{ccc}
A^{T} P+P A+G^{T} P G+\kappa \beta^{2} I & P D & P \\
* & 0 & 0 \\
* & * & -\kappa I
\end{array}\right) .
\end{gathered}
$$

So

$$
L V(x(t), t)-z^{T} Q z-2 z^{T} S v-v^{T} R v \leq \chi^{T}(t) \Theta^{\prime} \chi(t),
$$

where

$$
\begin{gathered}
\Theta^{\prime}=\left(\begin{array}{ccc}
\Sigma^{\prime} & P D-C^{T} S & P \\
* & -R & 0 \\
* & * & -\kappa I
\end{array}\right), \\
\Sigma^{\prime}=A^{T} P+P A+G^{T} P G+\kappa \beta^{2} I-C^{T} Q C .
\end{gathered}
$$

By Lemma 3, if (17) holds, then (11) holds. Since $R>0$, there exists a sufficient small $\alpha>0$ such that $R-\alpha I>0$. Together with (11), we have

$$
\Xi+\operatorname{diag}(0, \alpha I, 0)<0 .
$$

In the following section, we firstly consider the stability of NSISs in (10). Setting $v(t)=0$, by (11) and Lemma 4, we have

$$
L V(x(t), t)-z^{T} Q z-2 z^{T} S v-v^{T} R v \leq \alpha v^{T} v,
$$

which leads to

$$
\begin{aligned}
L V(x(t), t) & \leq x^{T}\left(C^{T} Q C-\delta Z\right) x \\
& \leq-\mu x^{T} P x=-\mu V(x(t), t),
\end{aligned}
$$

where $\mu=\lambda_{\min } P^{-1}\left(\delta Z-C^{T} Q C\right)>0$. 
Applying Dynkin formula and Grownwall-Bellman inequality, together with (21), for $t \in\left(t_{k-1}, t_{k}\right], k=1,2, \ldots$, we have

$$
E\{V(x(t), t)\} \leq V\left(x\left(t_{k-1}^{+}\right), t_{k-1}^{+}\right) \mathrm{e}^{-\mu\left(t-t_{k-1}\right)} .
$$

By Lemma 4 and (10), it follows that

$$
\begin{aligned}
V\left(x\left(t_{k-1}^{+}\right), t_{k-1}^{+}\right)= & x^{T}\left(t_{k-1}^{+}\right) P x\left(t_{k-1}^{+}\right) \\
= & x^{T}\left(t_{k-1}\right)\left[I+E_{k-1}+\bar{E}_{k-1}\right]^{T} \\
& \times P\left[I+E_{k-1}+\bar{E}_{k-1}\right] x\left(t_{k-1}\right) \\
\leq & \beta_{k-1} x^{T}\left(t_{k-1}\right) P x\left(t_{k-1}\right) \\
= & \beta_{k-1} V\left(x\left(t_{k-1}\right), t_{k-1}\right) .
\end{aligned}
$$

For $t \in\left(t_{0}, t_{1}\right]$, it follows from (22) and (23) that

$$
E\{V(x(t), t)\} \leq V\left(x\left(t_{0}\right), t_{0}^{+}\right) \mathrm{e}^{-\mu\left(t-t_{0}\right)},
$$

which leads to

$$
\begin{gathered}
E\left\{V\left(x\left(t_{1}\right), t_{1}\right)\right\} \leq V\left(x\left(t_{0}\right), t_{0}^{+}\right) \mathrm{e}^{-\mu\left(t_{1}-t_{0}\right)}, \\
E\left\{V\left(x\left(t_{1}\right), t_{1}^{+}\right)\right\} \leq \beta_{1} V\left(x\left(t_{0}\right), t_{0}^{+}\right) \mathrm{e}^{-\mu\left(t_{1}-t_{0}\right)} .
\end{gathered}
$$

Hence, for $t \in\left(t_{k-1}, t_{k}\right]$, it follows from (22)-(25) that

$$
\begin{aligned}
E\{V(x(t), t)\} \leq & V\left(x\left(t_{k-1}^{+}\right), t_{k-1}^{+}\right) \mathrm{e}^{-\mu\left(t-t_{k-1}\right)} \\
\leq & \beta_{k-1} V\left(x\left(t_{k-1}\right), t_{k-1}\right) \mathrm{e}^{-\mu\left(t-t_{k-1}\right)} \\
\leq & \beta_{k-1} V\left(x\left(t_{k-2}\right), t_{k-2}^{+}\right) \mathrm{e}^{-\mu\left(-t_{k-1}-t_{k-2}\right)} \\
& \times \mathrm{e}^{-\mu\left(t-t_{k-1}\right)} \leq \cdots \\
\leq & \beta_{k-1} \beta_{k-2} \cdots \beta_{1} V\left(x\left(t_{0}\right), t_{0}^{+}\right) \mathrm{e}^{-\mu\left(t-t_{0}\right)} .
\end{aligned}
$$

We know that

$$
\begin{gathered}
E \int_{t_{0}^{+}}^{t_{1}} V(x(t), t) \mathrm{d} t \\
\quad \leq E \int_{t_{0}^{+}}^{t_{1}} V\left(x\left(t_{0}\right), t_{0}^{+}\right) \mathrm{e}^{-\mu\left(t-t_{0}\right)} \mathrm{d} t \\
\quad=-\frac{1}{\mu} E\left\{V\left(x\left(t_{0}\right), t_{0}^{+}\right)\right\}\left[\mathrm{e}^{-\mu\left(t_{1}-t_{0}\right)}-1\right], \\
E \int_{t_{k-1}^{+}}^{t_{k}} V(x(t), t) \mathrm{d} t \\
\leq E \int_{t_{k-1}^{+}}^{t_{k}} V\left(x\left(t_{k-1}\right), t_{k-1}^{+}\right) \\
\times \mathrm{e}^{-\mu\left(t-t_{k-1}\right)} \mathrm{d} t
\end{gathered}
$$

$$
\begin{aligned}
= & -\frac{1}{\mu} E\left\{V\left(x\left(t_{k-1}\right), t_{k-1}^{+}\right)\right\} \\
& \times\left[\mathrm{e}^{-\mu\left(t_{k}-t_{k-1}\right)}-1\right] \\
\leq & -\frac{1}{\mu} E\left\{V\left(x\left(t_{k-1}\right), t_{k-1}^{+}\right)\right\} \\
& \times\left[\mathrm{e}^{-\mu\left(t_{k}-t_{k-1}\right)}-1\right] \\
\leq & -\frac{1}{\mu} \beta_{k-1} E\left\{V\left(x\left(t_{k-2}\right), t_{k-2}^{+}\right)\right\} \\
& \times\left[\mathrm{e}^{-\mu\left(t_{k-1}-t_{k-2}\right)}\right]\left[\mathrm{e}^{-\mu\left(t_{k}-t_{k-1}\right)}-1\right] \\
\leq & \cdots \leq-\frac{1}{\mu} \beta_{k-1} \beta_{k-2} \cdots \beta_{1} \\
& \times E\left\{V\left(x\left(t_{0}\right), t_{0}^{+}\right)\right\} \\
& \times\left[\mathrm{e}^{-\mu\left(t_{k}-t_{0}\right)}-\mathrm{e}^{-\mu\left(t_{k-1}-t_{0}\right)}\right] .
\end{aligned}
$$

In summary, we obtain that

$$
E \int_{t_{0}^{+}}^{T} V(x(t), t) \mathrm{d} t \leq-\frac{1}{\mu} E\left\{V\left(x\left(t_{0}\right), t_{0}^{+}\right)\right\} \theta
$$

where $\theta=\left[\mathrm{e}^{-\mu\left(t_{1}-t_{0}\right)}-1\right]+\beta_{1}\left[\mathrm{e}^{-\mu\left(t_{2}-t_{0}\right)}-\mathrm{e}^{-\mu\left(t_{1}-t_{0}\right)}\right]+\cdots+$ $\beta_{k} \beta_{k-1} \cdots \beta_{1}\left[\mathrm{e}^{-\mu\left(T-t_{0}\right)}-\mathrm{e}^{-\mu\left(t_{k}-t_{0}\right)}\right] ;$ let $\bar{\beta}=\max \left\{\beta_{1}, \beta_{2}, \ldots, \beta_{k}\right\}$, and we have

$$
\mathrm{e}^{-\mu\left(t_{1}-t_{0}\right)}-1-\mathrm{e}^{-\mu\left(t_{1}-t_{0}\right)} \frac{\bar{\beta}}{1-\bar{\beta}}<\theta<\mathrm{e}^{-\mu\left(t_{1}-t_{0}\right)}-1<0 .
$$

Therefore, we get

$$
E \int_{t_{0}^{+}}^{T} V(x(t), t) \mathrm{d} t \leq-\frac{1}{\mu} E\left\{V\left(x\left(t_{0}\right), t_{0}^{+}\right)\right\} \theta=-\frac{\theta}{\mu} x_{0}^{T} P x_{0} .
$$

Then, there exists a constant $c=-\theta \lambda_{\max }(P) / \mu \lambda_{\min }(P)>$ 0 , such that

$$
\lim _{T \rightarrow \infty}\left\{E \int_{t_{0}}^{T} V(x(t), t) \mathrm{d} t\right\} \leq c x_{0}^{T} x_{0}, \quad x_{0} \in R^{n}, t \geq t_{0} .
$$

Hence, the NSISs in (10) are stochastic stable. 
Secondly, we consider the $(Q, S, R)$-dissipativity of NSISs in (10). When $x\left(t_{0}\right)=0$, setting $v(t) \neq 0$, from (11) and (12), we have

$$
\begin{aligned}
& E \int_{t_{0}}^{T}\left[z^{T}(s) Q z(s)+2 z^{T}(s) S v(s)+v^{T}(s)(R-\alpha I) v(s)\right] \mathrm{d} s \\
&> E \int_{t_{0}}^{T} L V(x(s), s) \mathrm{d} s=E \int_{t_{0}}^{t_{1}} L V(x(s), s) \mathrm{d} s \\
&+E \int_{t_{1}^{+}}^{t_{2}} L V(x(s), s) \mathrm{d} s \\
&+\cdots+E \int_{t_{k}^{+}}^{T} L V(x(s), s) \mathrm{d} s \\
&= E V\left(x\left(t_{1}\right), t_{1}\right)-E V\left(x\left(t_{0}\right), t_{0}\right)+E V\left(x\left(t_{2}\right), t_{2}\right) \\
&-E V\left(x\left(t_{1}^{+}\right), t_{1}^{+}\right)+\cdots+E V(x(T), T) \\
&-E V\left(x\left(t_{k}^{+}\right), t_{k}^{+}\right) \\
& \geq \sum_{i=1}^{k}\left(1-\beta_{i}\right) E V\left(x\left(t_{i}\right), t_{i}\right)+E V(x(T), T) \\
&-E V\left(x\left(t_{0}\right), t_{0}\right) \geq E V(x(T), T)>0 .
\end{aligned}
$$

So it follows that (8) holds; thus the NSISs in (10) are strictly $(Q, S, R)$-dissipative according to Definition 2. The proof is complete.

Remark 6. From (12), we see that $\beta_{k}=\lambda_{\max }\left(P^{-1}(I+\right.$ $\left.\left.E_{k}+\bar{E}_{k}\right)^{T} P\left(I+E_{k}+\bar{E}_{k}\right)\right)<1$ is not a LMI; it is difficult to obtain the feasible solution, so by schur complement lemma, which is implied by a matrix inequality,

$$
\left(\begin{array}{cc}
P & \left(I+E_{k}+\bar{E}_{k}\right)^{T} \\
* & P^{-1}
\end{array}\right)>0 .
$$

4.2. State-Feedback Dissipative Control of NSISs. We are now ready to design the state-feedback controller $u(t)=K x(t)$ for the closed-loop NSISs in (1), and the NSISs in (1) can be rewritten as

$$
\begin{gathered}
\mathrm{d} x(t)=[(A+B K) x(t)+f(t, x(t))+D v(t)] \mathrm{d} t \\
+\sigma(t, x(t)) \mathrm{d} w(t), \quad t \in\left(t_{k-1}, t_{k}\right], \\
\Delta x\left(t_{k}^{+}\right)=\left(E_{k}+\bar{E}_{k}\right) x\left(t_{k}\right), \quad t=t_{k}, \\
z(t)=C x(t), \\
x\left(t_{0}^{+}\right)=x_{0} .
\end{gathered}
$$

Then, we have the following results.

Theorem 7. Given a real matrix $S$, a positive definite matrices $R>0$ and a negative semidefinite matrix $Q \leq 0$, if there exist a scalar $\delta>0$, and three matrices $X>0, Y$, and $\widetilde{Z}$, such that the following linear matrices inequalities (LMIs)

$$
\begin{gathered}
\Sigma=\left(\begin{array}{ccc}
\Sigma_{1} & D-X C^{T} S & \Sigma_{2} \\
* & -R & 0 \\
* & * & \Sigma_{3}
\end{array}\right)<0, \\
\left(\begin{array}{cc}
X & X\left(I+E_{k}\right)^{T}+Y_{k}^{T} \\
* & X
\end{array}\right)>0
\end{gathered}
$$

have a feasible solution, then the NSISs in (34) are stochastic stable and strictly $(Q, S, R)$-dissipative, the feedback gain is $K=$ $Y X^{-1}$, and the impulsive controller gain is $\bar{E}_{k}=Y_{k} X^{-1}$, where

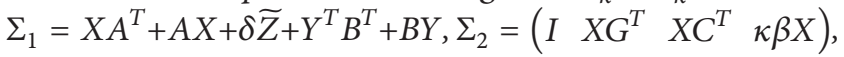
$\Sigma_{3}=\operatorname{diag}\left(-\kappa I,-X, Q^{-1},-\kappa I\right)$.

Proof. For NSISs in (34), applying Theorem 5 and Lemma 3, we have

$$
\left(\begin{array}{cccccc}
\Sigma_{1}^{\prime} & P D-C^{T} S & P & G^{T} & C^{T} & \kappa \beta I \\
* & -R & 0 & 0 & 0 & 0 \\
* & * & -\kappa I & 0 & 0 & 0 \\
* & * & * & -P^{-1} & 0 & 0 \\
* & * & * & * & Q^{-1} & 0 \\
* & * & * & * & * & -\kappa I
\end{array}\right)<0
$$

where $\Sigma_{1}^{\prime}=(A+B K)^{T} P+P(A+B K)+\delta Z$.

Applying the congruent transformation $T_{1}=\operatorname{diag}(X, I, I$, $I, I, I)$ and $T_{2}=\operatorname{diag}(X, I), X=P^{-1}$ to (37) and (33), respectively, introducing the linearization $\widetilde{Z}=X Z X$, and using $K X=Y, \bar{E}_{k} X=Y_{k}$, LMIs (35) and (36) hold. The proof is complete.

Remark 8. When $Q=-I, S=0$, and $R=\gamma^{2} I$, strictly $(Q, S, R)$-dissipative reduces to the $H_{\infty}$ performance level. When $Q=0, S=I$, and $R=\gamma I$, strictly $(Q, S, R)$-dissipative reduces to the strictly passivity.

Remark 9. If $\beta_{k}=\lambda_{\max }\left(P^{-1}\left(I+E_{k}\right)^{T} P\left(I+E_{k}\right)\right)<1$, then let $\bar{E}_{k}=0$, which means that there is no need for any impulsive controller for NSIS (1). If $\beta_{k}=\lambda_{\text {max }}\left(P^{-1}\left(I+E_{k}\right)^{T} P(I+\right.$ $\left.\left.E_{k}\right)\right)>1$, then choose appropriate $\bar{E}_{k}$ such that $\beta_{k}=$ $\lambda_{\text {max }}\left(P^{-1}\left(I+E_{k}+\bar{E}_{k}\right)^{T} P\left(I+E_{k}+\bar{E}_{k}\right)\right)<1$, which means that there is need of impulsive controller with gain $\bar{E}_{k}$ for NSISs in (1).

Corollary 10. If there exist a scalar $\delta>0$ and three matrices $X>0, Y$, and $\widetilde{Z}$, such that the inequalities

$$
\left(\begin{array}{cccccc}
\Sigma_{1} & D & I & X G^{T} & X C^{T} & \kappa \beta X \\
* & -\gamma^{2} I & 0 & 0 & 0 & 0 \\
* & * & -\kappa I & 0 & 0 & 0 \\
* & * & * & -X & 0 & 0 \\
* & * & * & * & -I & 0 \\
* & * & * & * & * & -\kappa I
\end{array}\right)<0
$$

and (36) have a feasible solution, then the NSISs in (34) are stochastic stable with $H_{\infty}$ performance level, and the feedback gain is $K=Y X^{-1}$. 
Corollary 11. If there exist a scalar $\delta>0$ and three matrices $X>0, Y$, and $\widetilde{Z}$, such that the inequalities

$$
\left(\begin{array}{ccccc}
\Sigma_{1} & D-X C^{T} & I & X G^{T} & \kappa \beta X \\
* & -\gamma I & 0 & 0 & 0 \\
* & * & -\kappa I & 0 & 0 \\
* & * & * & -X & 0 \\
* & * & * & * & -\kappa I
\end{array}\right)<0
$$

and (36) have a feasible solution, then the NSISs (34) are stochastic strictly passive, and the feedback gain is $K=Y X^{-1}$.

\section{Numerical Example with Simulation}

In this section, we will give an example to show the correctness of the derived results and the effectiveness of the proposed methods. Considering NSISs in (1), the system parameters are given as follows:

$$
\begin{aligned}
& A=\left(\begin{array}{cccc}
-1.5 & 1 & 0 & 1 \\
2 & 4 & 2.5 & 0 \\
-1 & -1.5 & 5 & 0 \\
0 & 0 & 2 & -5
\end{array}\right), \quad B=\left(\begin{array}{cc}
0 & 1 \\
-0.5 & 0 \\
-0.2 & 0 \\
0 & 0.2
\end{array}\right) \\
& C=\left(\begin{array}{cccc}
1 & 2 & 0 & 0 \\
0 & 0.5 & 0 & 0 \\
0 & 0 & 1 & 0 \\
0 & 0 & 0.2 & 1
\end{array}\right), \quad D=\left(\begin{array}{cccc}
0.4 & 0.2 & 0.1 & 0 \\
0.5 & 0.1 & 0.5 & 0.2 \\
0 & 0.1 & 0 & 1 \\
0.1 & 0 & 1 & 0.1
\end{array}\right) \\
& E=\left(\begin{array}{cccc}
-0.2 & 0 & 0 & 0 \\
0 & -0.1 & 0 & 0 \\
0 & 0 & -0.3 & 0 \\
0 & 0 & 0 & -0.2
\end{array}\right) \\
& f(x)=\left(\begin{array}{l}
0.1 x_{1} \sin x_{3} \\
0.2 x_{2} \cos x_{2} \\
0.1 x_{3} \sin x_{3} \\
0.3 x_{4} \cos x_{1}
\end{array}\right) \text {, } \\
& \sigma(t, x(t))=\left(\begin{array}{c}
0.5 x_{1} \sin x_{1} \\
\frac{t}{1+t} x_{2} \\
0.5 x_{3} \cos x_{3} \\
\frac{0.2 t}{t+2} x_{4}
\end{array}\right) \text {, } \\
& v(t)=\left(\begin{array}{c}
0.5 \sin 0.8 t \\
e^{-0.2 t} \cos t \\
0.3 \cos 0.5 t \\
e^{-t} \sin t
\end{array}\right), \quad-Q=R=S=I .
\end{aligned}
$$

From Figure 1, we can see that the uncontrolled NSISs in (1) are not stable; from (3)-(5), we can easily calculate that $\beta=$

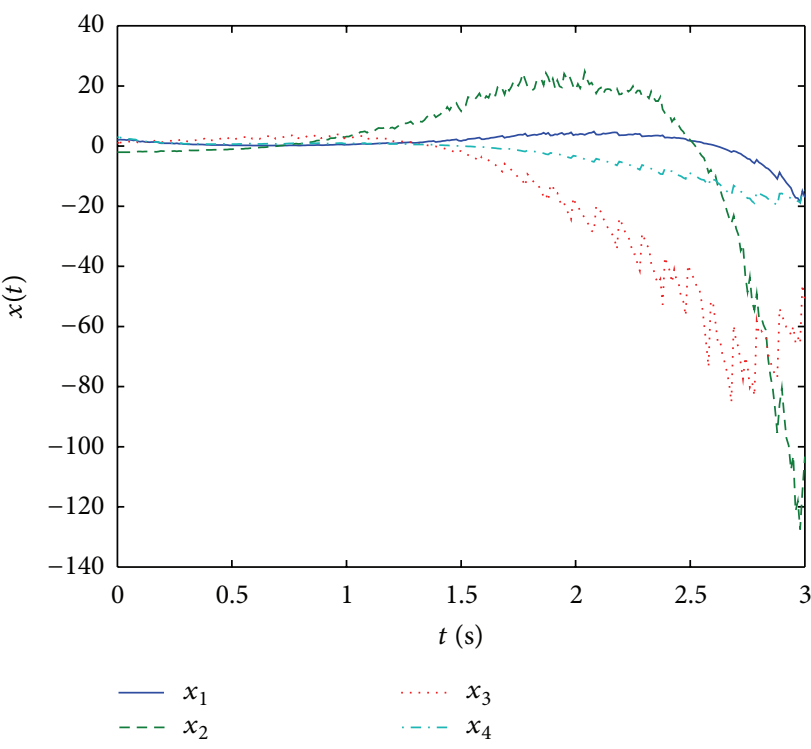

FIGURE 1: The state curves of the uncontrolled NSISs in (1) with $x(0)=\left(\begin{array}{llll}2 & -2 & 1 & 3\end{array}\right)^{T}$.

$0.3, G=\operatorname{diag}(0.5,1,0.5,0.2)$, and we choose $\kappa=1$. Applying Theorem 7 to this example with $\widetilde{Z}=0.2 I$, then we have

$$
\begin{gathered}
X=\left(\begin{array}{cccc}
218.4915 & -78.3446 & -28.6536 & 41.2314 \\
-78.3446 & 61.9663 & 23.7252 & -14.7112 \\
-28.6536 & 23.7252 & 9.2450 & -5.2351 \\
41.2314 & -14.7112 & -5.2351 & 11.9250
\end{array}\right), \\
Y=1.0 e+004 *\left(\begin{array}{cccc}
-0.0840 & 1.4332 & 0.5742 & -0.0115 \\
-1.0921 & 0.1494 & 0.0621 & -0.2110
\end{array}\right) .
\end{gathered}
$$

So the controller parameters can be calculated as follows:

$$
\begin{aligned}
& K=1.0 e+003 *\left(\begin{array}{cccc}
0.1421 & 0.2444 & 0.4278 & -0.0116 \\
-0.0786 & -0.4984 & 1.0753 & -0.0478
\end{array}\right), \\
& E_{k}=\left(\begin{array}{cccc}
-0.8 & 0 & 0 & 0 \\
0 & -0.9 & 0 & 0 \\
0 & 0 & -0.7 & 0 \\
0 & 0 & 0 & -0.8
\end{array}\right) .
\end{aligned}
$$

The state curves and the output curves of closed-loop NSISs in (1) can be seen in Figures 2 and 3.

From Figure 4, we can see that the closed-loop NSISs in (1) are strictly $(Q, S, R)$-dissipative, and Figure 5 shows the variation of $\alpha$ in $\Psi(v, z, T) \geq \alpha E \int_{0}^{T} v^{T}(t) v(t) \mathrm{d} t$, for all $T \geq 0$.

\section{Conclusions}

In this paper, the dissipativity analysis and control problems for a class of nonlinear stochastic impulsive systems (NSISs) have been investigated. The systems are subject to the nonlinear disturbance, stochastic disturbance, and impulsive effects, 


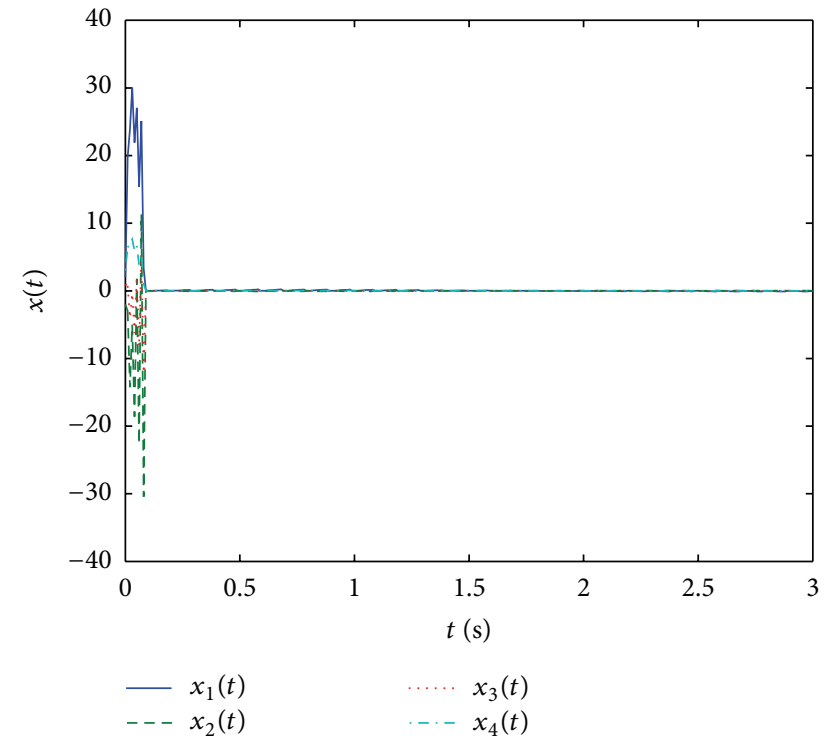

FIGURE 2: The state curves of the NSISs in (1) under state-feedback control and impulsive control with $x(0)=\left(\begin{array}{llll}2 & -2 & 1 & 3\end{array}\right)^{T}$.

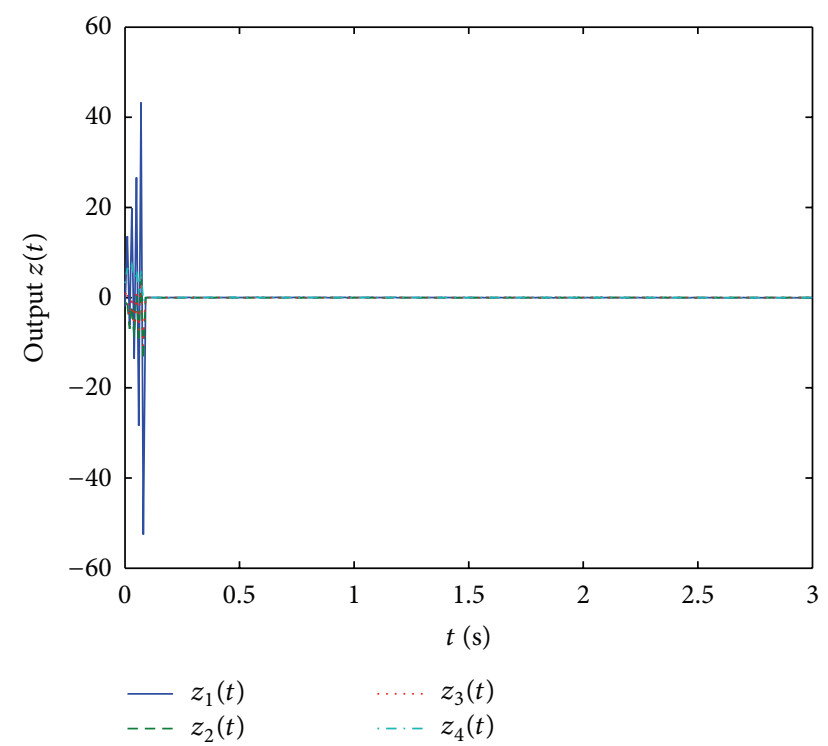

FIGURE 3: The output curves of the NSISs in (1) under state-feedback control and impulsive control with $x(0)=\left(\begin{array}{llll}2 & -2 & 1 & 3\end{array}\right)^{T}$.

which often exist in a wide variety of industrial processes and the sources of instability. Based on the dissipativity, the statefeedback controller and impulsive controller, such that the nonlinear stochastic impulsive systems are stochastic stable and strictly $(Q, S, R)$-dissipative, have been designed. The sufficient conditions have been obtained in terms of linear matrix inequalities (LMIs), and the given numerical example with simulation shows the correctness of the derived results and the effectiveness of the proposed method.

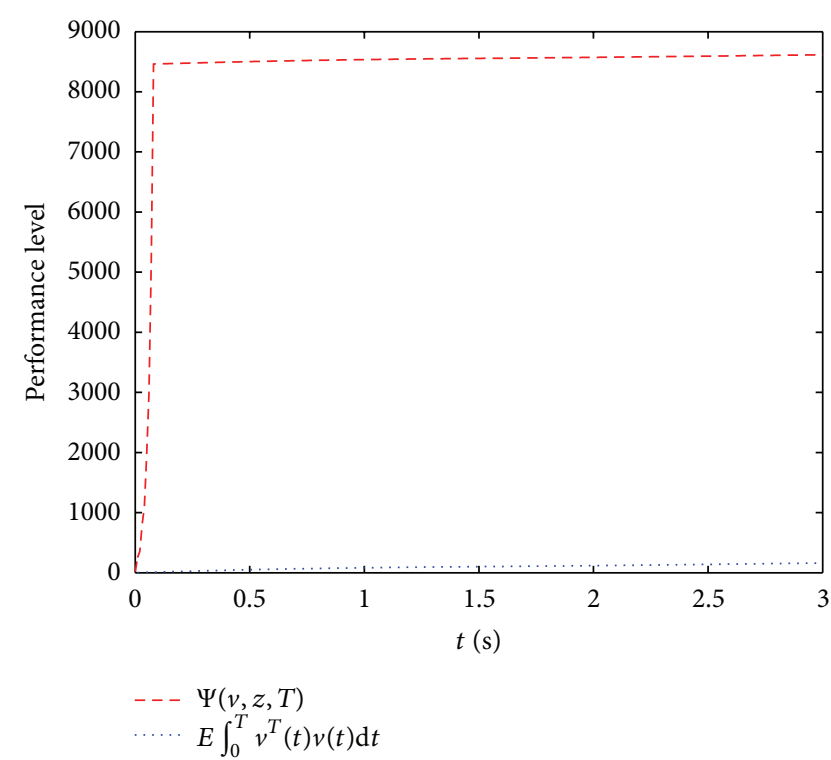

Figure 4: The curves of performance level $\Psi(v, z, T)$ and $E \int_{0}^{T} v^{T}(t) v(t) \mathrm{d} t$ of the NSISs in (1).

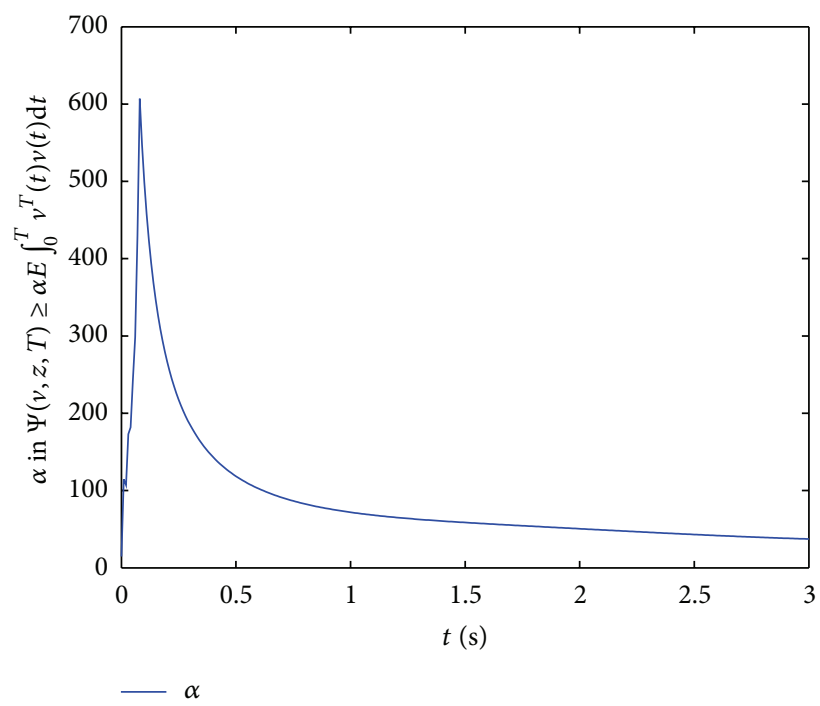

FIGURE 5: The variation curve of $\alpha$ in $\Psi(v, z, T) \geq \alpha E \int_{0}^{T} v^{T}(t) v(t) \mathrm{d} t$ in the NSISs in (1).

\section{Conflict of Interests}

The authors declare that there is no conflict of interests regarding the publication of this paper.

\section{Acknowledgments}

This work is supported by National Science Foundation of China (NSFC) under Grant nos. 61104127, 51079057, and 61134012, China Postdoctoral Science Foundation with Grant no. 2012M521428, and Hubei Province Key Laboratory of 
Systems Science in Metallurgical Process (Wuhan University of Science and Technology) under Grant no. Y201101.

\section{References}

[1] Y. Shen and J. Wang, "An improved algebraic criterion for global exponential stability of recurrent neural networks with timevarying delays," IEEE Transactions on Neural Networks, vol. 19, no. 3, pp. 528-531, 2008.

[2] V. Kolmanovskii and A. Myshkis, Introduction to the Theory and Applications of Functional-Differential Equations, vol. 463 of Mathematics and Its Applications, Kluwer Academic Publishers, Dordrecht, The Netherlands, 1999.

[3] Y. Shen and J. Wang, "Robustness analysis of global exponential stability of recurrent neural networks in the presence of time delays and random disturbances," IEEE Transactions on Neural Networks and Learning Systems, vol. 23, no. 1, pp. 87-96, 2012.

[4] G. Chen and Y. Shen, "Robust reliable $H_{\infty}$ control for nonlinear stochastic Markovian jump systems," Mathematical Problems in Engineering, vol. 2012, Article ID 431576, 16 pages, 2012.

[5] T. Li, W. X. Zheng, and C. Lin, "Delay-slope-dependent stability results of recurrent neural networks," IEEE Transactions on Neural Networks, vol. 22, no. 12, pp. 2138-2143, 2011.

[6] T. Li and Y. Zhang, "Fault detection and diagnosis for stochastic systems via output PDFs," Journal of the Franklin Institute, vol. 348, no. 6, pp. 1140-1152, 2011.

[7] B. Zhang, S. Xu, G. Zong, and Y. Zou, "Delay-dependent stabilization for stochastic fuzzy systems with time delays," Fuzzy Sets and Systems, vol. 158, no. 20, pp. 2238-2250, 2007.

[8] B. Zhang, S. Xu, and Y. Zou, "Output feedback stabilization for delayed large-scale stochastic systems with Markovian jumping parameters," Asian Journal of Control, vol. 11, no. 4, pp. 457-460, 2009.

[9] V. Lakshmikantham, D. D. Bainnov, and P. S. Simeonov, Theory of Impulsive Differential Equations, vol. 6 of Series in Modern Applied Mathematics, World Scientific Publishing, Teaneck, NJ, USA, 1989.

[10] D. D. Baĭnov and P. S. Simeonov, Systems with Impulse Effect: Stability, Theory and Applications, Ellis Horwood Series: Mathematics and Its Applications, Ellis Horwood, New York, NY, USA, 1989.

[11] C. Li, J. Sun, and R. Sun, "Stability analysis of a class of stochastic differential delay equations with nonlinear impulsive effects," Journal of the Franklin Institute, vol. 347, no. 7, pp. 1186-1198, 2010.

[12] B. Li, D. S. Li, and D. Y. Xu, "Stability analysis of impulsive stochastic delay differential equations with markovian switching," Journal of the Franklin Institute, vol. 350, no. 7, pp. 18481864, 2013.

[13] L. Pan and J. Cao, "Exponential stability of impulsive stochastic functional differential equations," Journal of Mathematical Analysis and Applications, vol. 382, no. 2, pp. 672-685, 2011.

[14] L. Shen, J. Sun, and Q. Wu, "Controllability of linear impulsive stochastic systems in Hilbert spaces," Automatica A, vol. 49, no. 4, pp. 1026-1030, 2013.

[15] W.-H. Chen and W. X. Zheng, "Robust stability and $H_{\infty}$-control of uncertain impulsive systems with time-delay," Automatica A, vol. 45, no. 1, pp. 109-117, 2009.

[16] X. Li and R. Rakkiyappan, "Impulse controller design for exponential synchronization of chaotic neural networks with mixed delays," Communications in Nonlinear Science and Numerical Simulation, vol. 18, no. 6, pp. 1515-1523, 2013.

[17] S. Xu and T. Chen, "Robust $H_{\infty}$ filtering for uncertain impulsive stochastic systems under sampled measurements," Automatica A, vol. 39, no. 3, pp. 509-516, 2003.

[18] J. C. Willems, "Dissipative dynamical systems. I. General theory," Archive for Rational Mechanics and Analysis, vol. 45, no. 5, pp. 321-351, 1972.

[19] D. Hill and P. Moylan, "The stability of nonlinear dissipative systems," IEEE Transactions on Automatic Control, vol. 21, no. 5, pp. 708-711, 1976.

[20] Z. Tan, Y. C. Soh, and L. Xie, "Dissipative control for linear discrete-time systems," Automatica A, vol. 35, no. 9, pp. 1557$1564,1999$.

[21] M. S. Mahmoud and A. Ismail, "Passivity and passification of time-delay systems," Journal of Mathematical Analysis and Applications, vol. 292, no. 1, pp. 247-258, 2004.

[22] M. S. Mahmoud, Y. Shi, and F. M. AL-Sunni, "Dissipativity analysis and synthesis of a class of nonlinear systems with timevarying delays," Journal of the Franklin Institute, vol. 346, no. 6, pp. 570-592, 2009.

[23] H. Zhang, H. Yan, and Q. Chen, "Stability and dissipative analysis for a class of stochastic system with time-delay," Journal of the Franklin Institute, vol. 347, no. 5, pp. 882-893, 2010.

[24] H. Zhang, Z.-H. Guan, and G. Feng, "Reliable dissipative control for stochastic impulsive systems," Automatica A, vol. 44, no. 4, pp. 1004-1010, 2008.

[25] S. Boyd, L. El Ghaoui, E. Feron, and V. Balakrishnan, Linear Matrix Inequalities in System and Control Theory, vol. 15 of SIAM Studies in Applied Mathematics, SIAM, Philadelphia, Pa, USA, 1994.

[26] L. Huang, Linear Algebra in Systems and Control Theory, Science Press, Beijing, China, 1984. 


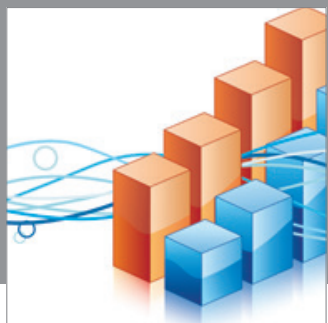

Advances in

Operations Research

mansans

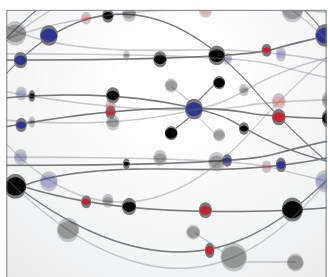

The Scientific World Journal
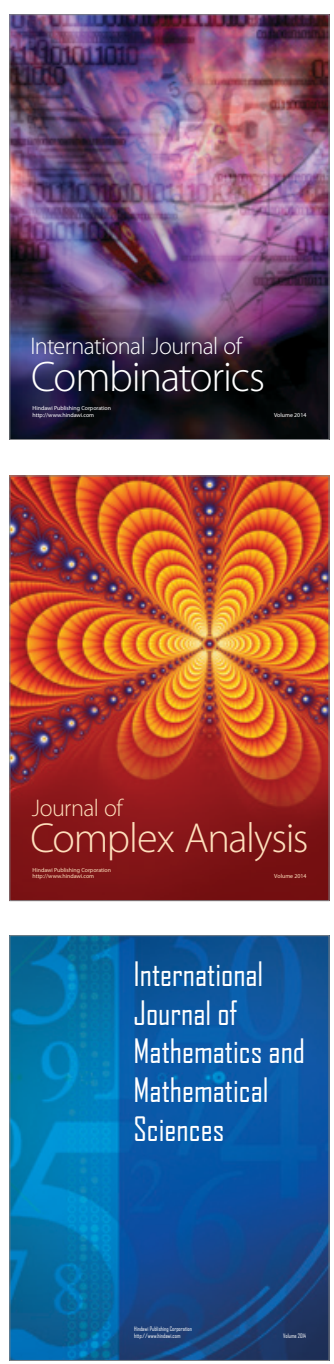
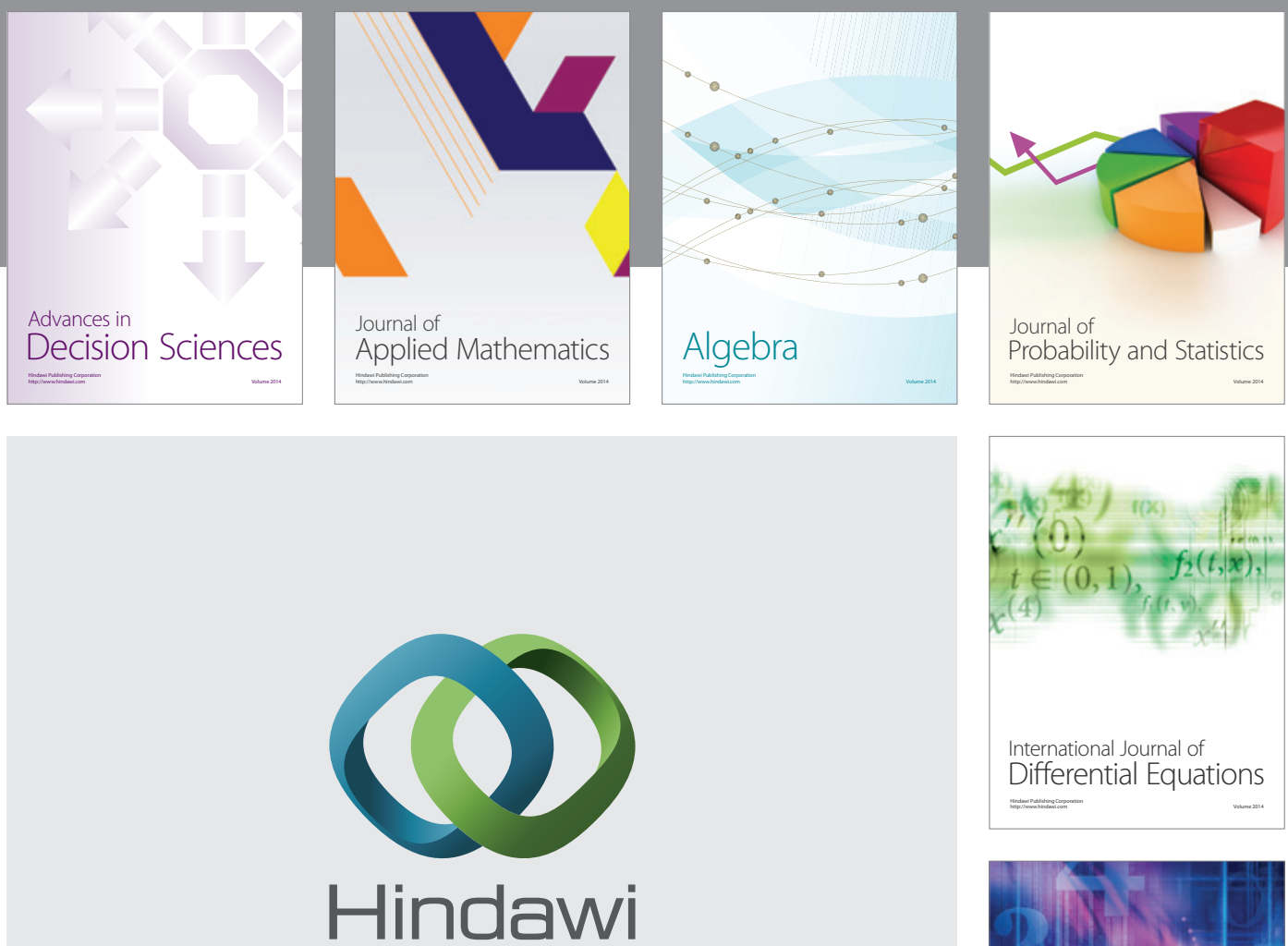

Submit your manuscripts at http://www.hindawi.com
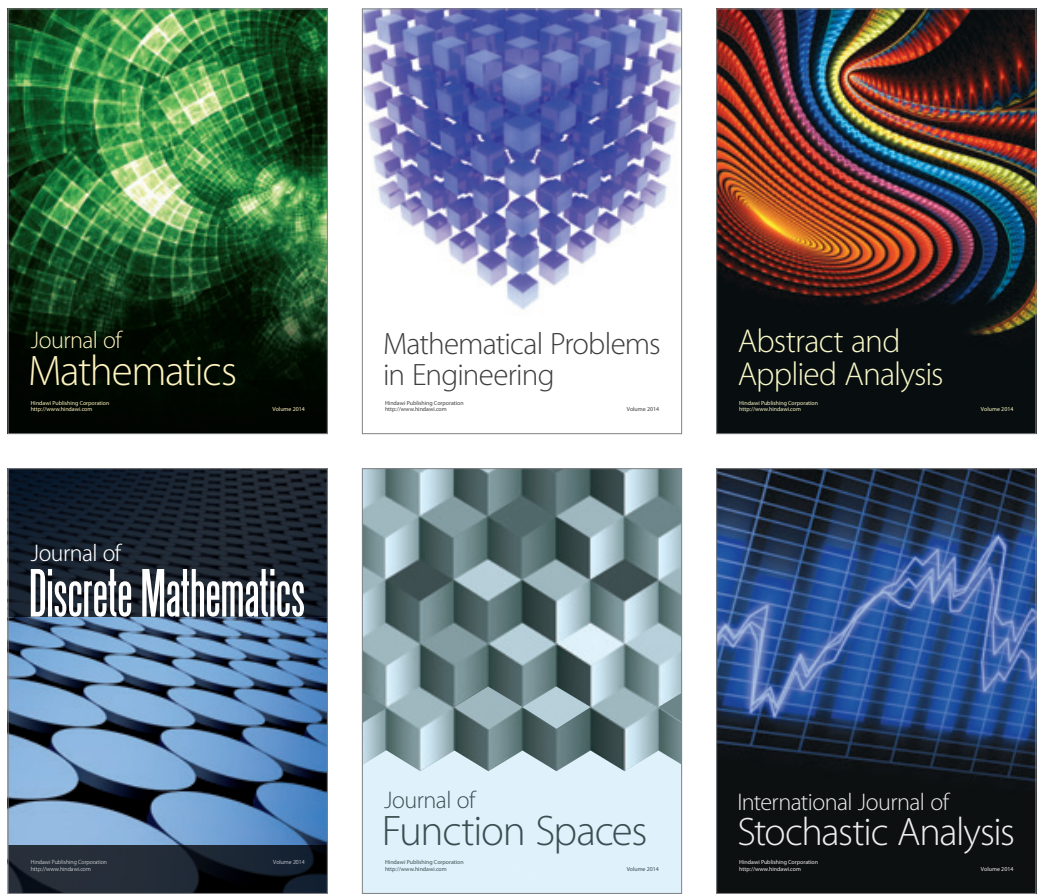

Journal of

Function Spaces

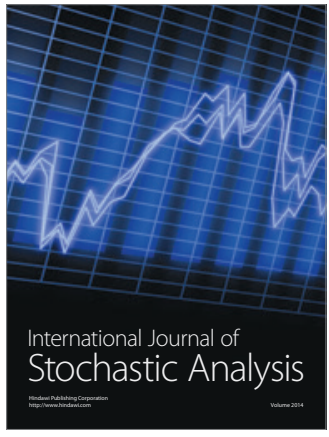

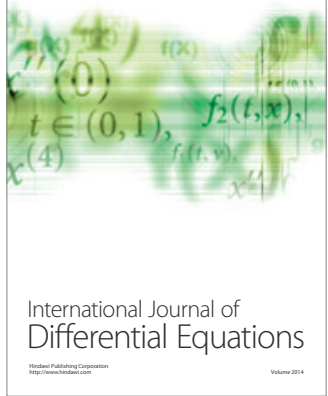
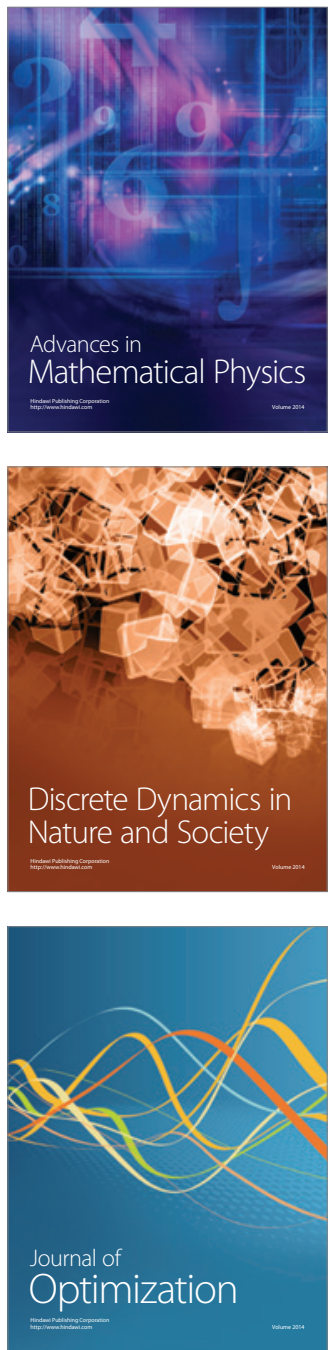